\title{
Low cost heads-up virtual reality (HUVR) with optical tracking and haptic feedback
}

\author{
Todd Margolis ${ }^{*}{ }^{*}$, Thomas A. DeFanti ${ }^{\mathrm{b}}$, Greg Dawe ${ }^{\mathrm{b}}$, Andrew Prudhomme ${ }^{\mathrm{b}}$, Jurgen P. Schulze ${ }^{\mathrm{b}}$, \\ Steve Cutchin ${ }^{\mathrm{c}}$ \\ ${ }^{a}$ CRCA, University of California, San Diego, 9500 Gilman Dr., La Jolla, CA, USA 92093-0037; \\ ${ }^{\mathrm{b}}$ Calit2, University of California, San Diego, 9500 Gilman Dr., La Jolla, CA, USA 92093-0436; \\ ${ }^{c}$ King Abdullah University of Science and Technology Visualization Laboratory, Thuwal, Saudi \\ Arabia
}

\begin{abstract}
Researchers at the University of California, San Diego, have created a new, relatively low-cost augmented reality system that enables users to touch the virtual environment they are immersed in.

The Heads-Up Virtual Reality device (HUVR) couples a consumer 3D HD flat screen TV with a half-silvered mirror to project any graphic image onto the user's hands and into the space surrounding them. With his or her head position optically tracked to generate the correct perspective view, the user maneuvers a force-feedback (haptic) device to interact with the 3D image, literally 'touching' the object's angles and contours as if it was a tangible physical object.

HUVR can be used for training and education in structural and mechanical engineering, archaeology and medicine as well as other tasks that require hand-eye coordination. One of the most unique characteristics of HUVR is that a user can place their hands inside of the virtual environment without occluding the 3D image. Built using open-source software and consumer level hardware, HUVR offers users a tactile experience in an immersive environment that is functional, affordable and scalable.
\end{abstract}

Keywords: Augmented reality, virtual reality, haptics, optical tracking, consumer 3D, affordable VR, collaborative virtual environments, scientific visualization

\section{INTRODUCTION}

The "classic" CAVE ${ }^{\mathrm{TM}}{ }^{1}$ is a cube-shaped virtual-reality (VR) room, typically $3 \mathrm{~m}-$ by- $3 \mathrm{~m}-$ by- $3 \mathrm{~m}$ in size, whose walls, floor and sometimes ceiling are entirely made of computer-projected screens. All participants wear active stereo glasses to see and interact with complex 3D objects. One participant wears a six degree-of-freedom location and orientation sensor called a tracker so that when he/she moves within the CAVE, correct viewer-centered perspective and surround stereo projections are produced quickly enough to give a strong sense of 3D visual immersion.

Projection-based VR systems, such as CAVEs, feature surround viewing (ideally fully-surround, but usefully at least $90^{\circ}$ in two dimensions such that users do not see the edges of the display). They offer stereo visuals. And, they track the user to provide the correct scene perspective rendering in a continuous manner. Viewer-centered perspective and surround viewing distinguish VR systems from 3D movies.

The classic CAVE was conceived and designed in 1991 by Tom DeFanti and Dan Sandin, who at the time were professors and co-directors of the Electronic Visualization Laboratory ${ }^{2}(\mathrm{EVL})$ at the University of Illinois at Chicago (UIC). Many students and colleagues over the years contributed to CAVE software and hardware development. ${ }^{3}$

*tmargolis@ucsd.edu; phone 1858 534-0184; fax 1858 534-7944; crca.ucsd.edu

Three-Dimensional Imaging, Interaction, and Measurement, edited by J. Angelo Beraldin, et al.,

Proc. of SPIE-IS\&T Electronic Imaging, SPIE Vol. 7864, 786417 · (c) 2011 SPIE-IS\&T

CCC code: 0277-786X/11/\$18 - doi: 10.1117/12.876584 
The first CAVE ${ }^{4}$ prototype was built in 1991, showed full scale $\left(3 \mathrm{~m}^{3}\right)$ in public at SIGGRAPH'92 ${ }^{5}$ and SC'92, and then CAVEs were built for the National Center for Supercomputing Applications, Argonne National Laboratory, and The Defense Advanced Research Projects Agency. In the past 17 years, hundreds of CAVEs and variants have been built in many countries. See Wikipedia Cave Automatic Virtual Environment ${ }^{6}$ for an excellent discussion of CAVEs and similar systems.

The first generation CAVE used active stereo (that is $96-160 \mathrm{fps}$ field-sequential images separated by glasses that synchronously blink left and right) to maintain separate images for the left and right eyes. Three-tube cathode ray tube (CRT) Electrohome ECP and then Marquee projectors (with special low-persistence green phosphor tubes) were used, one per $3 \mathrm{~m}^{2}$ screen, at a resolution of 1280 x $1024 @ 120 \mathrm{~Hz}$. The first CAVEs were relatively dim (the effect was like seeing color in bright moonlight), and somewhat stuttering (the networked Silicon Graphics, Inc. (SGI) workstations, one per projector, could maintain only about 8 updates of a very modest 3-D perspective scene per second, insufficient for smooth animation). Ascension, Inc. Flock of Birds electromagnetic tethered trackers were used to poll the 6 degreeof-freedom (DOF) position of the user's head and hand. There were three rear-projected walls and a down projected floor, which gave a then novel complete feeling of room-sized immersion. The screen frame was made of non-magnetic steel to decrease interference with the tracker, and the screen was a grey flexible membrane screen stretched over cables in 2 corners. About $85 \%$ of the cost of the first generation CAVE was in the 5 SGI Crimson workstations, later the 4output 8-processor SGI Onyx.

A second-generation CAVE was developed by EVL in $2001^{7}$, featuring Christie Mirage DLP 1280x1024 projectors that were 7 times brighter than the Electrohomes of the first generation, although 5 times the cost. Users' color perception got much better because of the brighter projectors delivering adequate light to their eyes' color receptors. This system also used active stereo at $60 \mathrm{~Hz} /$ eye (the projectors update at $120 \mathrm{~Hz}$ ) and could, with the SGI Reality Engine, get $\sim 25$ graphic scene updates per second, a $3 \mathrm{x}$ improvement over the first-generation SGI Crimsons, resulting in much smoother motion. For this CAVE version, still sold, about $60 \%$ of the cost was in the SGI 8-processor shared-memory cluster. A third generation CAVE using passive polarization projection, called the StarCAVE, was built in $2007 .^{8}$

The same CAVE Wikipedia entry accurately describes EVL's 1998 ImmersaDesk system as follows:

The biggest issue that researchers are faced with when it comes to the CAVE is size and cost. Researchers have realized this and have come up with a derivative of the CAVE system, called ImmersaDesk. With the ImmersaDesk, the user looks at one projection screen instead of being completely blocked out from the outside world, as is the case with the original CAVE. The idea behind the ImmersaDesk is that it is a single screen placed on a 45-degree angle so that the person using the machine has the opportunity to look forward and downward. The screen is 4' X 5', so it is wide enough to give the user the width that they need to obtain the proper 3-D experience. The 3-D images come out by using the same glasses as were used in the CAVE. This system uses sonic hand tracking and head tracking, so the system still uses a computer to process the users' movements.

This system is much more affordable and practical than the original CAVE system for some obvious reasons. First, one does not need to create a "room inside of a room". That is to say that one does not need to place the ImmersaDesk inside of a pitch-black room that is large enough to accommodate it. One projector is needed instead of four, and only one projection screen. One does not need a computer as expensive or with the same capabilities that are necessary with the original CAVE. Another thing that makes the ImmersaDesk attractive is the fact that since it was derived from the original CAVE, it is compatible with all of the CAVE's software packages and also with all of the CAVE's libraries and interfaces.

A further development came with the PARIS system', which shared the ImmersaDesk's basic layout, but featured a semi-transparent mirror so that one could see one's hands and interactive devices through the mirror as well as the VR graphics projected correctly in space on them. One PARIS system was built twelve years ago and it still works, but, like the ImmersaDesk, it cost more than $\$ 100,000$.

HUVR builds upon this previous work on the PARIS system. HUVR is less than $1 / 10$ the cost of PARIS and has significantly higher graphics throughput and better color and contrast. With several consumer flat screen 3D TVs now on the market, we realize with HUVR a new means for building low cost VR systems. 




Figure 1. Artist rendering of the original PARIS system using an Electrohome projector to supply the stereoscopic imagery.

\subsection{Consumer Devices}

Capitalizing on the wave of recent successful Hollywood 3D films, manufacturers of flat screen TVs have been adding stereoscopic displays to their lineups for slightly more cost than their 'mono' counterparts. Driven largely by the multibillion dollar computer game industry, basic force feedback interfaces ("rumble" controllers which can shake and vibrate) are now ubiquitous and advanced systems are much more affordable. This gives users the ability to have a wide range of interactive interfaces with virtual environments. HUVR also builds upon advances in the tele-communications industry with computer vision. Smart phones with built-in cameras and force-feedback are bringing us closer to a persistent augmented reality and consumer video tele-conferencing systems like Skype have made web cameras more widespread. Developments in open-source computer vision libraries have made a broad array of complex algorithms for image processing and object tracking accessible to enable new forms of real-time interactivity. HUVR uses commodity webcams to provide head tracking which is both high quality and affordable, enabling an untethered and interactive experience. Building upon the growth of global high-speed fiber networks, tele-collaboration between multiple HUVR systems is easily achieved. Virtual reality software frameworks such as COVISE $^{3}$ have built-in collaborative functionality. As long as VR systems are connected via a network, they can be linked on an application level to allow for multi-user collaboration. We successfully created a collaborative session between two HUVR systems in our laboratory, but these systems could be located anywhere in world as long as they are connected to the internet. This allows application scenarios such as remote medical training, the training of technicians who need to repair complex machinery, or any other application domain the HUVR is useful for. In a collaborative session, the users typically share the same virtual space with the same data, but can position themselves independently or deliberately in the same spot, whichever is more desirable.

\subsection{Application Domains}

Potential applications of HUVR include visualizing and manipulating a 3-D image of a person's brain taken from an MRI, training for finding defects in precious metals, or analyzing artifacts too fragile or precious to be handled physically. Additionally, the applications discussed in "Advanced Applications of Virtual Reality"10 as well as recent demonstrations of the StarCAVE ${ }^{11}$ can be extended to the HUVR system. 


\section{DESIGN OF SYSTEM}

Our primary aim in designing HUVR was to create an affordable immersive augmented reality system that incorporates real-time stereoscopic 3D images, haptics and head tracking. As shown in Figure 2, a 3D display is mounted horizontally to an 8020 frame above a desk in front of the user. The images are reflected onto a semi-transparent mirror back to the user who wears specialized eyewear synchronized to the display to see alternating left/right eye images. A haptics controller is mounted on the table to touch the objects in the virtual environment. Directional lighting under the mirror reveals the user's hands holding the virtual objects. The user's head position is tracked via cameras and retro-reflective markers on the eyewear.

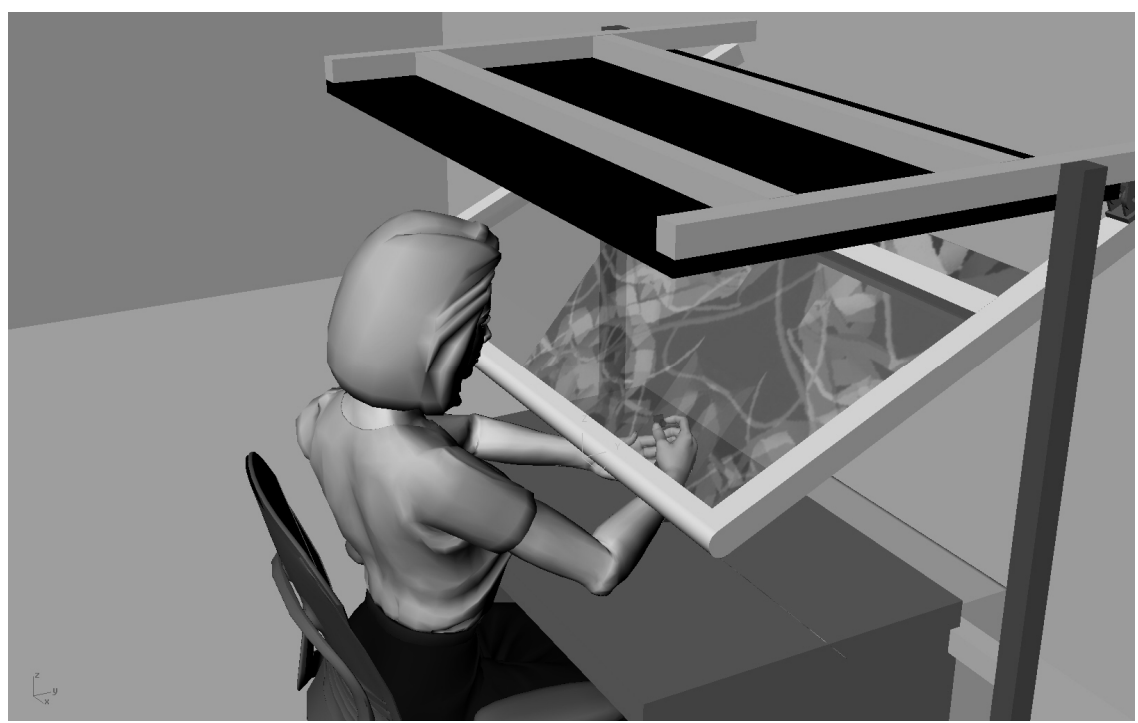

Figure 2. Basic prototype CAD drawing of HUVR

\subsection{Stereoscopic Screens}

Three different types of displays were evaluated which can be classified into two main categories of stereoscopy - active and passive. The JVC GD-463D1I0U LDC Monitor uses a micropole passive (left/right eye spatially separated) system which works well for tiling multiple displays together. Passive stereo glasses are significantly less expensive compared to active eyewear. The JVC model alternates horizontal lines for each eye thereby reducing the overall resolution of the display from HD to half HD. We ruled out the JVC because the passive system had significant problems with polarization and the mirrors.

We decided to use two displays (the Samsung 55C7000 LCD and Panasonic TC-P50VT25 Plasma displays) which both use active (left/right eye temporally sequenced) stereoscopic systems. Both require the use of active eyewear which is more costly due to the need for internal electronics to manage synchronization and switching each eye on and off.

\subsection{Mirrors}

The earlier PARIS employed a semi-transparent mirror fabricated by laminating a mylar based self-adhesive commercial window tinting material onto a transparent cast acrylic sheet. The assembly was installed to place the reflective element (the first surface) between the screen and user. This material is available with various levels of transmission/reflectivity. It is typically labeled using the percentage of light transmitted assuming the surface is normal to the light path. Typical spec: $50 \%$ transmitted, $\sim 45 \%$ reflective, $\sim 5 \%$ absorbed, or $80 \%$ trans, $\sim 15 \%$ ref, $\sim 5 \%$ absorbed. Note that a transmissive mirror's apparent reflectivity increases (transmission decreases) proportionally as the mirror is rotated from normal to the light path, as it is in our case. As transmission increases, the brightness of the reflected image is decreased; more of the illumination light scattered from within the augmented volume is transmitted to the user and more of the second surface reflected image is noticed.

Given the abundant brightness of the current generation flat panels tested, $25 \%$ to $75 \%$ transmission would be acceptable. Two brands of aluminum coated "tint" product were used to build sample mirrors; both laminated assemblies 
introduced objectionable color moiré patterns. Suspecting the adhesive layer was introducing a bi-fringent filter effect, a pre-coated acrylic transmissive mirror (security mirror) sheet was acquired and tested. This product eliminated the color moiré, but introduces some geometric distortions (likely due to non-uniform coating thickness). Further research will be required to identify the optimal mirror.

A quick test with a photo light meter indicates $\sim 75 \%$ of the panel's light is reflected to the user, so the coating would likely be specified as $50 \%$ transmissive.

\subsection{Haptics}

Basic force-feedback "rumble" controllers have emerged as a mainstream gaming commodity. These provide a very modest sense of "touch" or in many cases are more of a reaction to an event i.e. gunshot or explosion. These generally retail within the $\$ 30-\$ 100$ range. The next step up in game controllers in the $\$ 200-\$ 500$ range use similar low cost motors offering rough/weak feedback by providing some resistance. In the $\$ 2000-\$ 10000$ range spatial resolution and more powerful feedback is provided.

We decided to use a mid-range devices - Falcon and the Omni by SensAble who make very precise high quality haptics. We would prefer to use SensAble's Desktop version, but it is quite expensive and would double the cost of the other components combined.

The Falcon has a very limited range of motion to touch virtual objects. To overcome this, we used a scaling factor to feel larger objects. This has the unfortunate side effect of dis-locating the virtual haptic sphere from the physical haptic sphere.

\subsection{Tracking}

High-end motion capture technologies have become a reliable standard tool for the vast majority of motion pictures as well as video games. Many of these cameras use embedded micro-controllers to achieve high speed acquisition and processing causing them to be very high quality, but out of reach for the consumer market.

The ubiquity of commodity technologies such as webcams, smart phones and surveillance cameras has led to more accessible price points as well as increased quality. Open source libraries such as OpenCV bring together advanced techniques for image processing and object tracking.

We have taken two approaches to head tracking - the first is with custom software using OpenCV and an $\$ 11$ surveillance camera. The second option is using a Vicon ${ }^{12}$ two camera Bonita system with Vicon's Tracker software.

Both systems use VRCO's trackd to communicate tracker data with the graphics host.

\subsection{Ergonomics}

HUVR differs from most VR systems in that the image plane is not located on a physical surface. Because we are reflecting the stereoscopic imagery from the 3D display onto an angled mirror, the image plane is located at twice the angle of the mirror from the display, This forces the user's eyes to focus on an imaginary surface behind the mirror.

Given the small region that exists to "touch" objects, the haptic volume needed to be co-located with the center of the image plane to achieve visual accommodation. One of the most compelling achievements of HUVR is that a virtual object in space can be held without occlusion. This is possible because images are 'projected' onto the mirror, but a user's hands are still visible behind the mirror. This effect is impossible to achieve with traditional rear-projection virtual reality systems and head mounted displays.

\subsection{Visualization Software}

\subsection{1 $\operatorname{COVISE}^{3}$ (Collaborative Visualization and Simulation Environment) ${ }^{13}$}

COVISE was originally developed at the High Performance Computing Center Stuttgart (HLRS), and has been commercialized by the Stuttgart-based VISENSO GmbH. It is a toolkit to integrate several stages of a scientific or technical application such as grid-generation, simulation, data import, post-processing, and visualization. Each step is implemented as a module. Using a visual user interface, these modules can be connected to a data flow network. Each of 
the computational and I/O modules in this workflow can reside on a different computer. This allows distributing the work load among different machines. For instance, the pre- and post-processing modules can run on a visualization server, while the simulation runs on a remote supercomputer. The display modules can run on the workstation of a user, or on a visualization cluster driving a multiscreen visualization environment. COVISE's virtual reality rendering module, OpenCOVER can run on a variety of interactive displays and environments. It can even be used on a single computer with a mouse, but then the user cannot take advantage of its immersive capabilities. OpenCOVER is ideally run on tracked stereo environment, using 3D pointing devices. OpenCOVER uses the OpenSceneGraph API for its 3D rendering, which is an object-oriented framework on top of OpenGL. OpenCOVER is an open interface, in that the application programmer can write plug-in modules in $\mathrm{C}++$ to create customized virtual reality applications, using COVISE's support of a large variety of virtual reality input and output devices, as well as interaction handling and network communication algorithms. The latter allow OpenCOVER to link multiple virtual environments together over the Internet, allowing for collaborative sessions with multiple end points. These collaborative sessions are managed by the Virtual Reality Request Broker (VRB), a stand-alone software application which comes with COVISE, to which all participants connect. The VRB allows individual participants to connect and disconnect to a collaborative session at any point, without disrupting the connections of the other participants.

\subsubsection{OpenSceneGraph (OSG) ${ }^{14}$}

OSG is a popular, open source, multi-platform computer graphics scene graph API. It is written in C++ and provides a well-designed class hierarchy for the implementation of real-time computer graphics and virtual reality projects. It builds on OpenGL and abstracts OpenGL's state machine to higher level data structures which allow the creation of complex graphics scenes without being hindered by the fine granularity of OpenGL. OSG also automatically optimizes its scene graph for maximum rendering performance wherever possible, it provides object based culling, intersection testing which is useful for user interaction, $2 \mathrm{D}$ and $3 \mathrm{D}$ file loaders, and it supports shaders to allow for more realistic rendering.

\subsubsection{Haptics Libraries}

HAPI is a C++ open source cross-platform haptics library. The majority of our haptics implementation uses this library. It was convenient to use as it also uses a scene graph similar to what we had already implemented for rendering visuals. This library was used exclusively to implement haptics for the Falcon.

For the SensAble Omni, we needed to incorporate the SensAble Technologies OpenHaptics Toolkit. This programming API has been designed for use with the SensAble PHANTOM force-feedback haptic devices. The API provides C++ functions to process positional and button data from the haptic device, and it allows the programmer to control force and torque of the PHANTOM's handle. The library is available for Windows and Linux. It consists of multiple software layers on top of the hardware driver, to accommodate programmers with different requirements for the level of device control needed.

\subsection{Tele-Collaboration}

Because HUVR is ideally suited for multi-user applications, it was important to demonstrate multiple forms of telecollaboration. There are essentially two modes of sharing control and environments. In "loose" mode, each user is able to freely navigate and interact with the world independently. In "tight" mode, one user at a time can control navigation. While in "tight" mode there are two ways of orienting the users. The first is where both users see the world from the same perspective. This has the advantage of allowing users to point out objects of interest to each other (e.g. touching a crack in an artifact in front of them). The second is where each of the users have their own fixed perspective next to each other. This configuration can match a physical layout of multiple HUVR systems in the same space (e.g. two surgeons standing across from each other over a body).

\section{IMPLEMENTATION}

Given the various design goals, two versions of HUVR were created to meet to multiple budget and application needs as well as to demonstrate the tele-collaborative potential of the system. As shown in figure 3, the system on the left is a 'low cost" option using the most affordable equipment. The system on the right is still quite affordable, but uses a more capable haptic system and tracking system for a higher quality experience. 


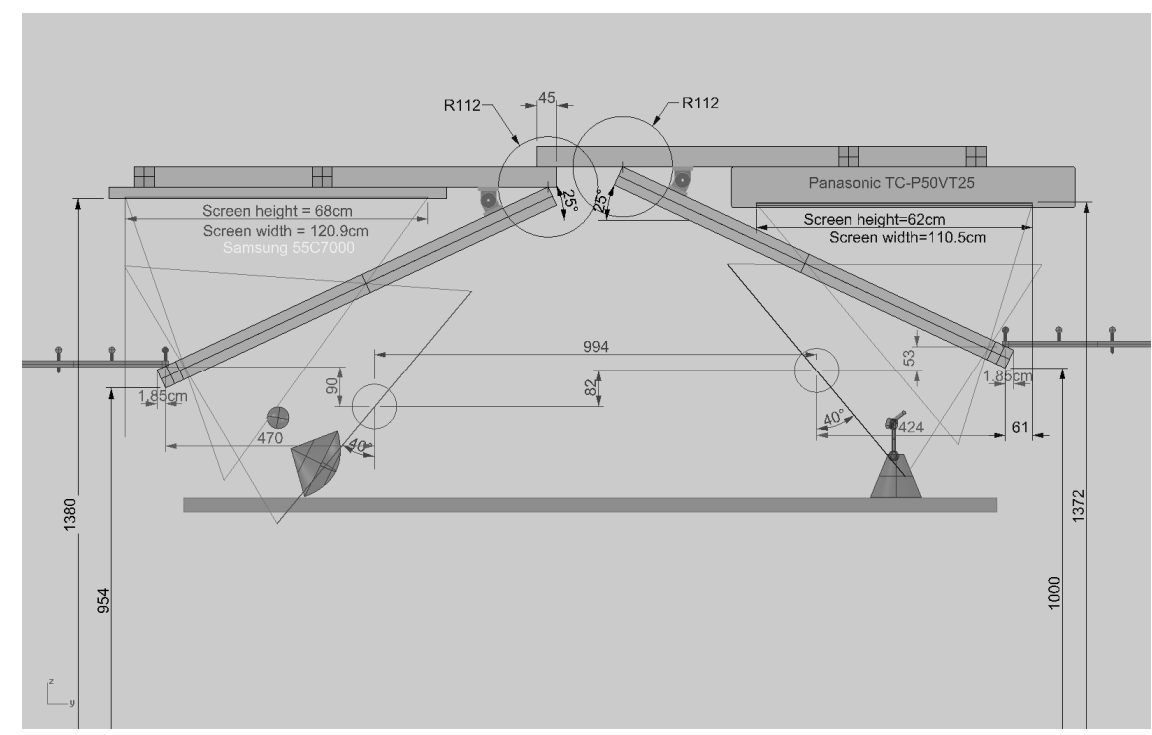

Figure 3. Dimensional drawing to help collocate origins for tracking, COVISE \& haptics.

\subsection{General issues}

There were several issues with the IR sync signal for switching glasses. First, the operating volume for the glasses is rather small and given the IR emitter is located on the bottom corner of the screen (which is the rear left when mounted) the range for receiving sync from the display feels rather limited. We decided to add a small piece of aluminized mylar to help reflect the sync signal towards the tracking space. We also found there was a conflict between the Samsung IR sync signal and the Vicon Bonita near-IR camera illuminators. The illuminators emanated a rather wide spectrum light which tended to confuse the receivers on the glasses. The solution to this was to mount a small piece of a Ratan visible light cut filter over the receiver on the glasses.

\subsection{Low Cost}

The system on the left of figure 3 uses a Samsung LCD display, Novint Falcon haptic system and IR webcam.

The Samsung LCD display provides a clear image and can quickly switch between side-by-side 3D and mono mode.

Head tracking for this HUVR version is accomplished with custom computer vision and a low-cost consumer webcam. Given the HUVR system would potentially be setup in uncontrolled lighting environments, we chose to use an IR filter with IR illuminators to minimize ambient lighting. Initially, we used a Logitech Quickcam 9000 which we needed to disassemble to remove an internal IR filter. A significant amount of extra IR illuminators were required to sufficiently flood the camera's view with light to sustain positive matches and minimize jitter. However, it proved to be unstable with our custom mount and wide-angle lens. We then identified another USB 2.0 webcam from Kinamax which seems primarily targeted towards surveillance with model number WCM-6LNV 6-LED Infrared Night-Vision 1.3 MegaPixel. This camera can be purchased for around \$15 and comes with an internal IR filter and built-in IR LEDs.

We use the OpenCV C++ library in Fedora linux to perform facial detection and optical flow. Using the OpenCV library in $\mathrm{C}++$ proved quite powerful. Our first attempt was to use Haar's cascades ${ }^{15}$ to perform real-time facial detection. This worked well with the built-in cascade files to find faces in well-lit environments. However, in our setup users were required to wear active eyewear which proved to confuse the detection algorithm. Given these problems we decided to add a second pass filter using Lucas-Kanade ${ }^{16}$ optical flow. The tracker uses the location of the last-found head location to search a thresholded image for the two retro-reflective markers.

The Novint Falcon attaches to the host computer via a USB. As described above, integrating haptics with OpenCOVER was achieved using the HAPI haptics library. First a haptics device must be created which will then connect to the first found device. Then a haptics renderer must be initialized and passed to the haptics device. Finally a shape and surface must be created and added to the device. 


\subsection{High Quality}

The system on the right in figure 3 uses a Panasonic Plasma display, SensAble Omni haptic system and Vicon Bonita cameras for head tracking.

The Panasonic Plasma provides an equally high quality image. Switching between 3D and mono mode is slightly more difficult than with the Samsung display.

Vicon calibration on HUVR is complicated due to the small area and reflective environment. The standard 5 marker 'wand' with $25 \mathrm{~mm}$ balls is too big for HUVR so one must use a smaller wand. We had a 3 marker 'wand' with $15 \mathrm{~mm}$ balls from our motion capture studio which worked quite well. However, we still used the 5 marker wand for setting the origin.

The SensAble Omni connects to the host computer via Firewire 400. For our application to be able to use the Omni instead of the Falcon, we only needed to add a few device drivers and include the OpenHaptics library with our plugin.

\section{RESULTS}

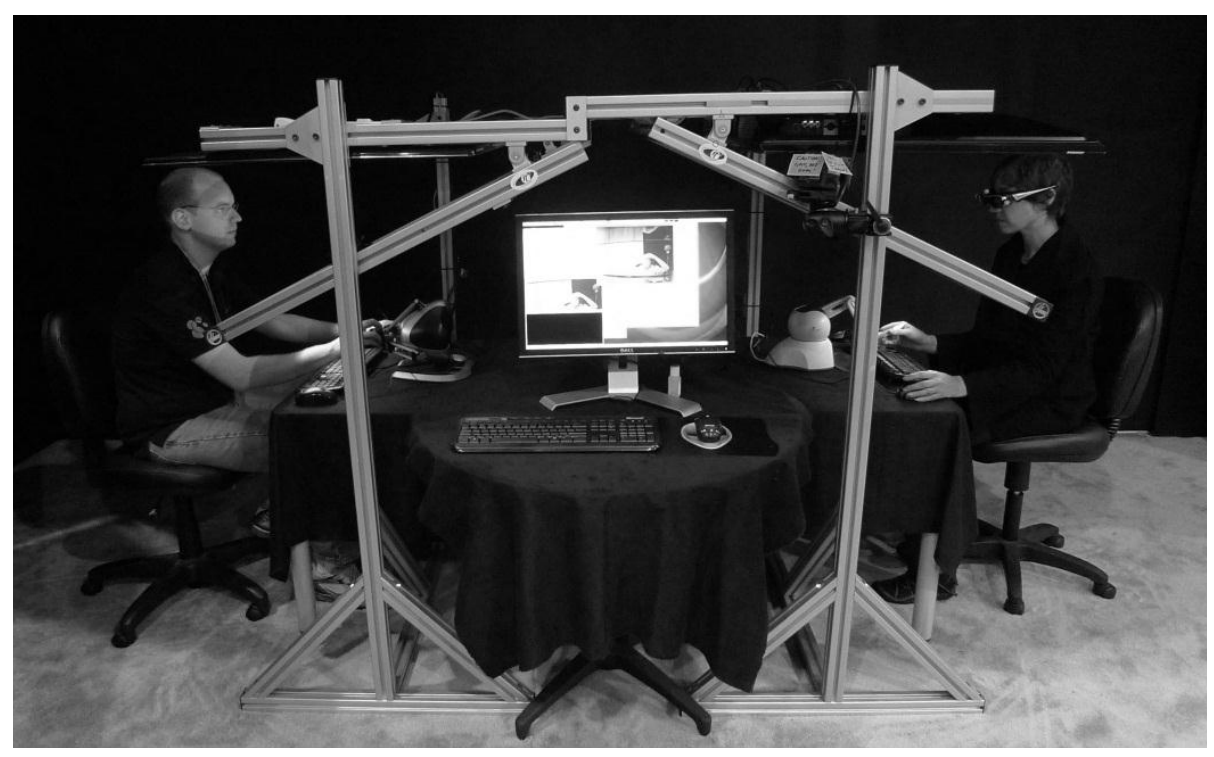

Figure 4. HUVR systems at SC10

\subsection{Image Quality}

The color moiré problem with the final mirror selection appears to have almost completely disappeared. We suspect that the moiré may still exist, but is now on a scale aligned with the pixel grid. Experienced users have also reported an overall improved viewing condition which may be attributed to less ghosting.

The Panasonic plasma display exhibited noise in solid areas of color in the image that could be distracting in certain imagery but was usually not noticeable when viewing dynamic stereo imagery or when the user was head tracked.

\subsection{Tracking Quality}

The Vicon system is robust, reliable and accurate. Physical setup, calibration and configuration become more streamlined after training. One must be careful that the cameras are not accidentally bumped as small movements will require a re-calibration. The system is highly scalable to extend the tracking volume and can easily track multiple objects at once. Generally, accuracy depends on the number of cameras and the quality of the calibration. In many cases the more markers you attach to the rigid object you want to track, the more successful the results will be. We have found that Tracker reports $6 \mathrm{DOF}(\mathrm{x} / \mathrm{y} / \mathrm{z}$ position and roll/pitch/yaw orientation) with very low jitter and latency. The only con 
to the Vicon Tracker solution is that the cost is still relatively high. For example, the 2 camera Bonita system with Tracker license is twice the cost of the rest of the equipment combined.

The OpenCV tracker works well under controlled situations. A lot of time was dedicated to using Haar's cascades to perform facial detection. It would work very well for detecting one person, but not so effective with two or more people. Additionally if the room environment changed, there was always the possibility for finding false positives. So the decision was made to add the optical flow technique with markers on the glasses. This greatly improved tracking accuracy \& reliability.

\subsection{Haptic Quality}

We have found that most users who have never worked with a haptics system before prefer the Falcon, however those that have some experience with haptics prefer the Omni. We believe that this may be due to the fact that the Falcon appears to have a more elastic response which gives a clear indication of when a user has encountered a virtual surface. However, the Omni provides a more concrete force which can confuse users to think that perhaps they are just hitting the maximum range of the haptic volume. Some users have also reported that the Falcon 'ball' is easier to grip than the Omni stylus. Most users agree that the larger haptic volume of the Omni is preferable to the more confined volume of the Falcon.

\subsection{Tele-Collaboration}

We found in general that using loose mode is the most expedient way to share a virtual environment. This is due to the fact that all participants can freely navigate the space without forcing the other participants to be tethered to their movements. One unfortunate side effect of this is that it is quite easy to lose the other tele-participants. out of view or to get hidden behind virtual walls.

\section{CONCLUSIONS AND FURTHER WORK}

In November of 2010, we will officially premiere HUVR at SC10 in New Orleans. We look forward to hearing the reaction from the Supercomputing community, making refinements and reporting on these ongoing developments.

\subsection{Minimize ghosting \& color moirés}

We plan to test new mirror surfaces to continue to optimize the image quality and transparency.

\subsection{Improve OpenCV Tracking to 6DOF}

While our custom OpenCV tracking works well for providing a subset (left/right and up/down) of all 6 degrees of freedom, we look forward to adding another camera to improve functionality, reliability and accuracy.

\subsection{Extend tracking for hand gesture recognition}

In addition to tracking the head position for proper viewer center perspective, we have always known how important it is to track hand positions as well. However, just tracking the hand location and the vector it is pointing, is no longer enough today for most complex VR interactions. We have already begun testing fully articulated hand/finger tracking with gesture recognition. We have experimented with using the MIT colored glove tracking with basic gesture recognition ${ }^{17}$. This system has the ability to represent two hands in a virtual environment and trigger events similar to mouse clicks when a user strikes certain poses. We have also been speaking with Oblong to discuss ways to integrate their rich g-speak spatial operating environment ${ }^{18}$ with our augmented realities to add an intuitive complex set of gestures to control navigation and interaction.

\subsection{Test all tele-collaborative options}

Given all of the possible configuration needs for tele-collaboration, a simple script system would provide a quick and easy way to rapidly test and deploy different options for sharing virtual environments between multiple users. 


\section{ACKNOWLEDGEMENTS}

This publication is based in part on work supported by Award No. US 2008-107, made by King Abdullah University of Science and Technology, in particular, the development and construction of the HUVRs.

VRCO/MechDyne, Vicon, Tracy Cornish

\section{REFERENCES}

[1] Cruz-Neira, C., Sandin, D., DeFanti, T., et.al., "The CAVE," Communications of the ACM, 35(6), 64-72 (1992)

[2] Electronic Visualization Laboratory, University of Illinois Chicago, http://www.evl.uic.edu

[3] DeFanti, T., et. al., “The Future of the CAVE”, Cent. Eur. J. Eng., 1(1) , (2011)

[4] The name CAVE ${ }^{\mathrm{TM}}$ was coined by DeFanti in 1992 for the VR room being built at the Electronic Visualization Laboratory (EVL), University of Illinois at Chicago (UIC), which was subsequently commercialized by the company that is now Mechdyne, Corporation.

[5] Michael Deering of Sun Microsystems, Inc. exhibited a single-user 3-wall similar system called the Portal at SIGGRAPH'92 < Deering, M., "High resolution virtual reality", ACM SIGGRAPH Computer Graphics, 26(2), 195-202 (1992)>. The 3-wall+floor CAVE at SIGGRAPH'92 allowed multiple users.

[6] CAVE Automatic Virtual Environment, , http://en.wikipedia.org/wiki/Cave_Automatic_Virtual_Environment

[7] Mirage 5000 CAVE, ,http://www.evl.uic.edu/pape/CAVE/DLP/

[8] DeFanti, T., Dawe, G., Sandin, D., Schulze, J., et.al., "The StarCave, a third-generation cave and virtual reality Optiportal," Future Generation Computer Systems/The International Journal of Grid Computing: Theory, Methods and Applications, Elsevier B.V., Vol 25, Issue 2, Nov. 2008.

[9] Johnson, A., Sandin, D., Dawe, G., Defanti, T., Pape, D., Qiu, Z., Thongrong, S. and Plepys D., "Developing the PARIS: Using the CAVE to Prototype a New VR Display," CDROM Proc. IPT (2000)

[10] Schulze, J., Kim, H. S., Weber, P., Prudhomme, A. Bohn, R. E., Seracini, M., DeFanti, T. "Advanced Applications of Virtual Reality", Book chapter in preparation for Advances in Computers, Editor: Marvin Zelkowitz, (2011)

[11] Further examples of the breath of StarCAVE application:

http://www.calit2.net/newsroom/article.php?id=1584

http://www.youtube.com/calit2\#p/search/0/pFTfZ0VtzIU

http://www.calit2.net/newsroom/article.php?id=1584

http://www.youtube.com/calit2\#p/search/0/pFTfZ0VtzIU (NexCAVE display of 3D model of rebar layout for new span of the San Francisco Bay Bridge

http://www.youtube.com/calit2\#p/search/1/9tEkHz97yNUhttp://www.youtube.com/calit2\#p/search/1/9tEkHz97yN $\mathrm{U}$ (NexCAVE display of 3D model of Calit2 headquarters building at UC San Diego) 
http://www.youtube.com/calit2\#p/search/2/AEjJYepZ_P8http://www.youtube.com/calit2\#p/search/2/AEjJYepZ_P 8 (NexCAVE display of archeological dig site model)

http://www.youtube.com/calit2\#p/search/3/Ze5IprHtC5chttp://www.youtube.com/calit2\#p/search/3/Ze5IprHtC5c (NexCAVE with 3D model of de-salination plant designed at the National University of Singapore)

http://www.youtube.com/calit2\#p/search/4/cQP_YhtAeyQhttp://www.youtube.com/calit2\#p/search/4/cQP_YhtAey $\mathrm{Q}$ (NexCAVE exploration of 3D model for the holy shrine at Mecca)

[http://www.youtube.com/calit2\#p/search/5/u96M4OhwIgA[http://www.youtube.com/calit2\#p/search/5/u96M4Oh wIgA (NexCAVE Exploration of Jordan Archaeological Excavation Site)

[12] Vicon Tracker, ,http://www.vicon.com

[13] Visual Engineering Solutions, ,www.visenso.de

[14] OpenSceneGraph, ,www.openscenegraph.org

[15] Viola, Jones, "Rapid object detection using boosted cascade of simple features", Computer Vision and Pattern Recognition, (2001)

[16] Lucas, B. D., Kanade, T., "An iterative image registration technique with an application to stereo vision," Proceedings of Imaging Understanding Workshop, 121-130 (1981)

[17] Wang, R. Y., Popovic, J., "Real-Time Hand-Tracking with a Color Glove", ACM Transaction on Graphics, 28(3):1-8, (2009)

[18] Oblong G-speak, ,http://www.oblong.com 